\title{
VARIATION OF PUPIL SIZE WITH CHANGE IN THE ANGLE AT WHICH THE LIGHT STIMULUS STRIKES THE RETINA*
}

BY

\section{K. H. SPRING and W. S. StILES}

THE possibility of a variation in pupil size depending on the part of the natural pupil through which the light from the external field enters the eye has not been examined hitherto. Such a variation might occur for two reasons : (a) since the apparent brightness of an illuminated field is reduced if the rays enter near the edge rather than near the centre of the pupil (directional sensitivity of the retina + ), a slightly larger pupil might be expected in the former case; $(b)$ if, as is possible, the protective action of the constricted pupil consists not so much in reducing the total light flux as in excluding rays which would otherwise strike the retina obliquely, such rays might prove more effective in closing the pupil than rays incident normally. The pupil would then be smaller for rays entering near the edge. While variations from these causes are likely to be smaller than the effects of changes in field brightness and field area, they would be of considerable theoretical interest and would have a bearing on the efficient design and use of optical instruments. In the present work, carried out at the request of the Director of Scientific Research, Admiralty, measurements of the pupil were made under conditions specially arranged to reveal any change of size with change in the point of entry of the light in the pupil.

To obtain the greatest possible separation between rays entering centrally and near the edge, the pupil must be fully dilated. On the other hand it is the pupil of moderate size which is most sensitive to changes in the conditions of illumination. Both requirements were met by exposing the illuminated field to one eye only, the pupil of this eye being kept fully dilated with a mydriatic, and by determining by flash photography the variations in size of the free (unmydriasised) pupil of the other eye which was kept in the dark. It was confirmed that a given light stimulus entering either eye affected both pupils about equally and that mydriasis of one pupil did not interfere with the reaction of the other.

In the two sets of main measurements, made on 12 subjects, the

* Communicated by the National Physical Laboratory. Received for publication April 15, 1948.

† For a review of the work done up to 1939 on this property of the retina (StilesCrawford effect) see Stiles, Science Progress, Vol. XXXIII, p. 176, 1939. 
rays from a Maxwellian field of $52^{\circ}$ diameter (white light) were focused in the plane of the mydriasised pupil of the left eye, the cross-section of the beam in this plane being approximately $0.5 \mathrm{~mm}$. Thus the free right pupil was the one photographed. The principle of the apparatus is made clear by the sketch in Fig. 1 (not to scale). The subject's head was kept rigid by requiring him to bite on a dental impression which was clamped to a massive lathe sliderest mounted on the table carrying the optical apparatus. The 3dimensional adjustment of the slide-rest enabled the subject's eyes to be brought into precisely the correct position with respect to the apparatus. Also by means of the transverse horizontal adjustment the head could be moved so that the rays from the $52^{\circ}$ field entered the dilated pupil of the left eye at any point on a horizontal diameter. For photographing the right pupil, the infra-red light from a 500 watt Pointolite was focused on the eye and exposed by a shutter for $1 / 20$ th sec. A second, synchronised shutter exposed the camera lens. Kodak Extra-Rapid Infra-red plates were used.

In the first series of main measurements the beam entered the left eye centrally. The variation of the apparent pupil size (apparent horizontal diameter) of the right eye was determined for field intensities from zero to $10^{5}$ luxons (photons)* The results for the 12 subjects (average age 29, range 25-46) are shown in Fig. 2, each curve being based on 2 or 3 sets of measurements. In each set, the observations. began after the subject had been in the dark for about $15 \mathrm{~min}$. Thereafter, the right pupil was photographed after $3 \mathrm{~min}$. exposure of the left eye to each of an increasing series ot field brightnesses. The left pupil was not mydriasised. There are very large individual differences and it may be noted that for several subjects the decrease in pupil size with increase in field brightness appears to occur in two stages. The results of these subjects are chiefly responsible for the slight irregularity in the mean curve (Fig. 3) at an intensity in the neighbourhood of $10^{0.5}$ luxons (marked $\mathrm{X}$ ). The second curve in Fig. 3 gives the mean results obtained in an unpublished investigation by the Admiralty Research Laboratory in which the subjects (52, average age 24 ) viewed, binocularly and with the natural pupil, a field of $50^{\circ}$ diameter. This curve also shows a slight irregularity (marked Y) but at a much lower intensity, 10-0.9 luxons.

To investigate the variation of apparent pupil size with point of entry a fixed field brightness in the range indicated in Fig. 3 was used. The left pupil was mydriasised, and the right photographed

* The photon is a unit of retinal illumination, and is the illumination corresponding to an external field of brightness one candle per square metre seen through a pupil of $1 \mathrm{sq}$. mm. area. Following Blottiau, the term "luxon" is now used to avoid confusion with the "photon" of quantum theory. 


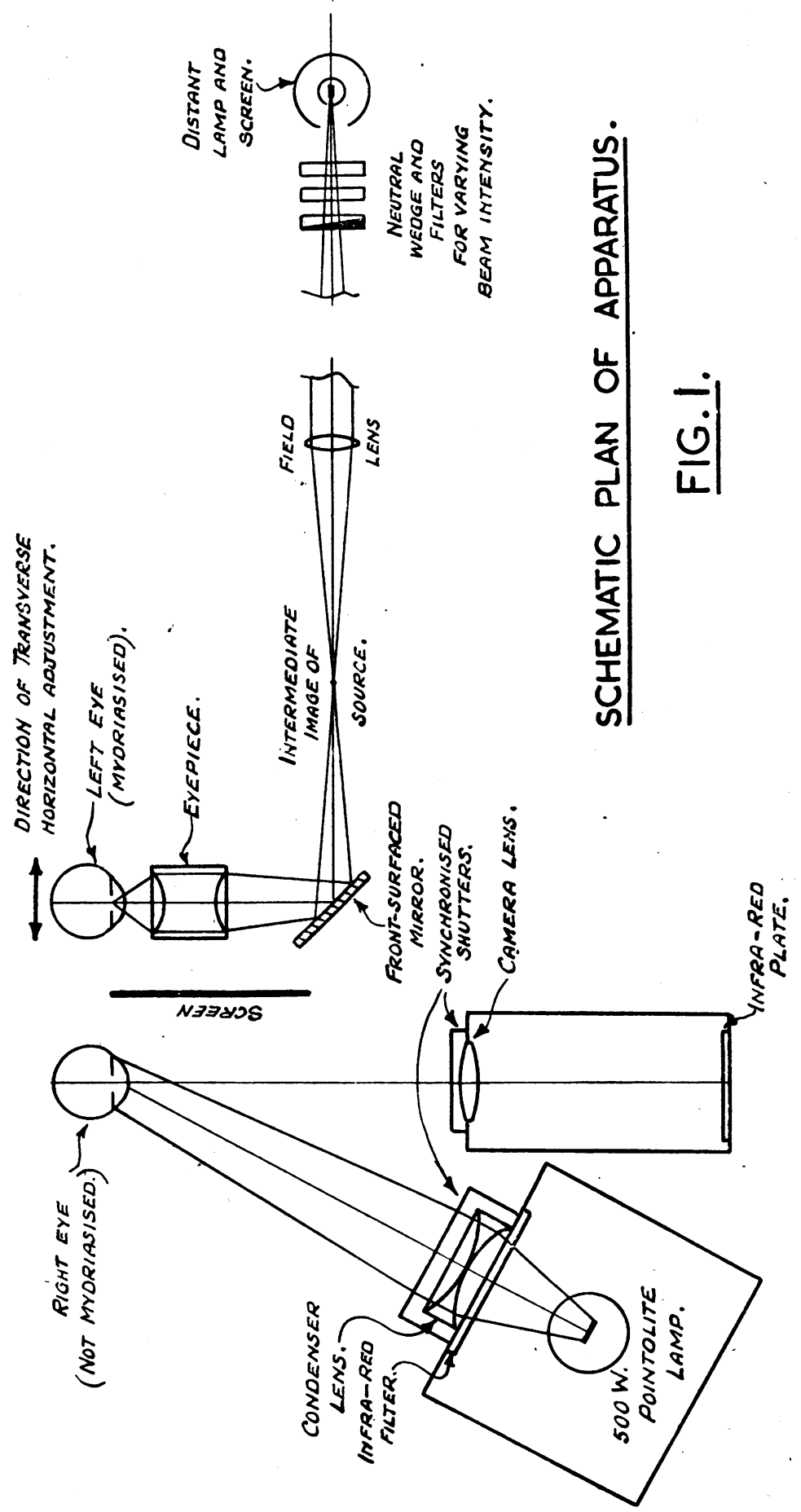


Variation of Pupil Size

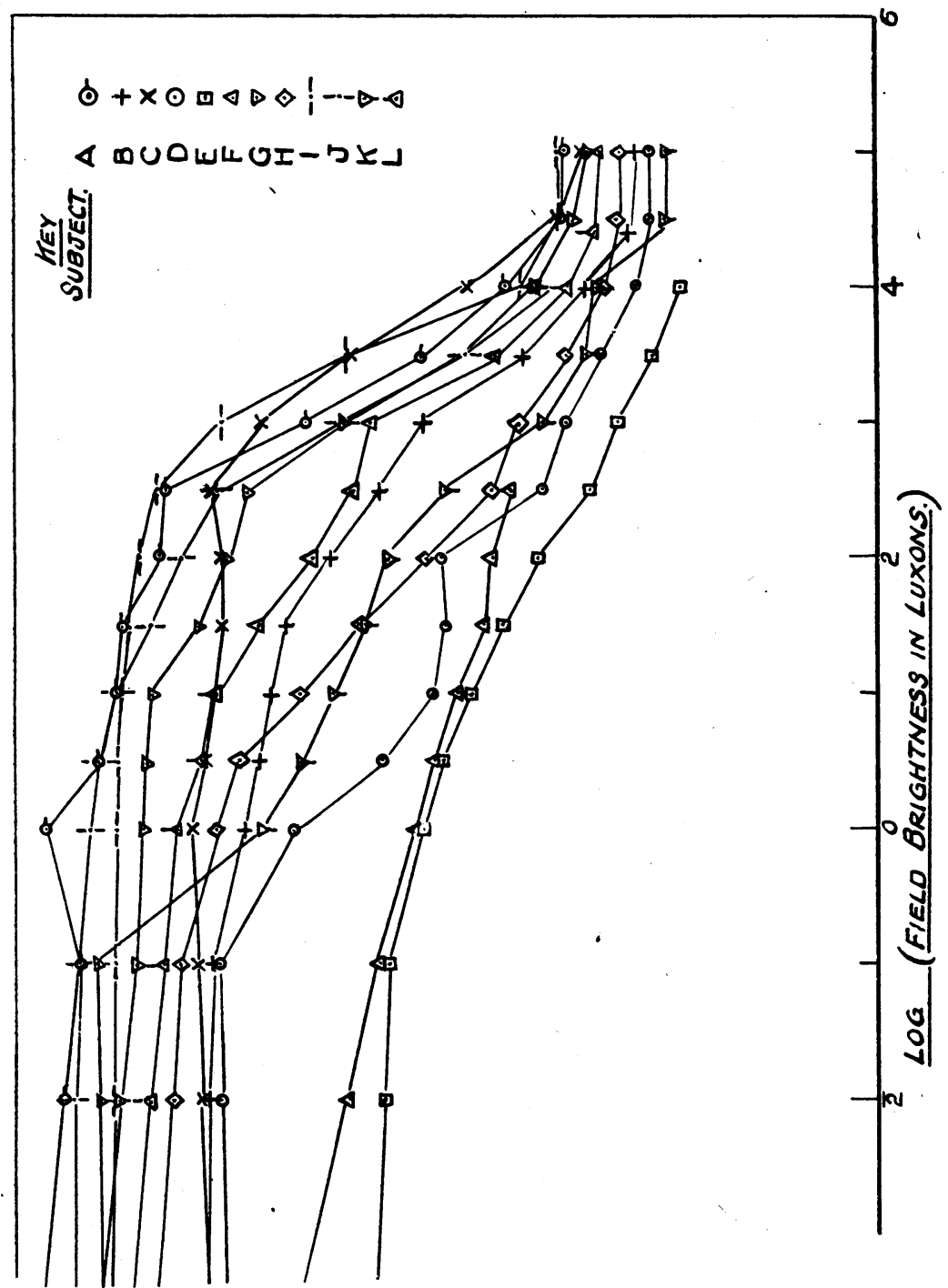

PUPIL SIZE - FIELD BRIGHTNESS CURVES FOR 12 SUBJECTS (52॰ FIELD.)

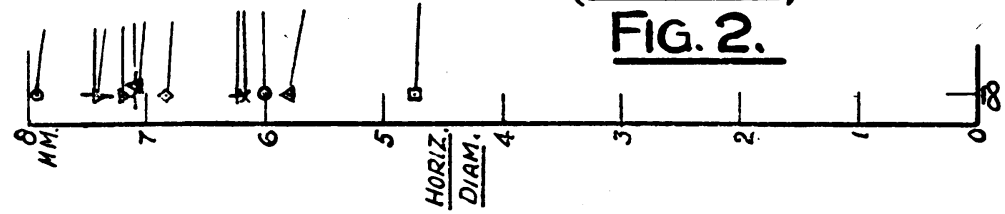


after the usual 3 minute preadaptation. The individual results (Fig. 4) are the means derived from 3 or 4 traverses of the pupil. A few of the more regular graphs show a tendency for the pupil to dilate slightly for eccentric entry. For the mean curve (Fig. 5) the change is very small and may not be significant : there is an increase from a diameter of $3.75 \mathrm{~mm}$. for central entry to $4.1 \mathrm{~mm}$. for entry at $3 \mathrm{~mm}$. nasal and $4.0 \mathrm{~mm}$. for entry at $3 \mathrm{~mm}$. temporal.

As the point of entry is traversed across the pupil the apparent brightness of the field changes. For foveal vision, the apparent brightness is reduced by a factor $\eta$, given approximately by the formula $\log \eta=-0.05 \mathrm{~d}^{2}$ where $\mathrm{d}$ is the distance of the point of entry from the pupil centre, expressed in $\mathrm{mm}$. (Stiles, 1939). Taking for each subject the gradient of the curve of pupil diameter against $\log$ (field brightness) (Fig. 2), at the brightness used for the traverse measurements (Fig. 4), the change of pupil size with position of the point of entry can be calculated, on the assumption that it arises solely from the change in apparent field brightness predicted by the above formula. The mean curve calculated in this way is shown in Fig. 5. It is evident that the observed variation is less than that calculated. The calculation assumes in effect that at the brightness levels in question only the retinal cones are concerned in determining the pupil size. The retinal rods have nearly the same sensitivity for all points of entry of a light stimulus and if they are participating in the control of the pupil the variation of pupil size with point of entry may be expected to be less.

Comparison for each subject of the pupil size obtained in the traverse measurements (Fig. 4) with the pupil size at the same brightness in the brightness level measurements (Fig. 2) shows that the mydriasis of the left pupil in the former case has not affected the control of the right pupil by the light stimulus entering the left eye. The difference in pupil size for the two cases averaged over the 12 subjects is $0 \cdot 13 \mathrm{~mm}$.

It may be concluded from the present measurements $(a)$ that rays entering near the edge of the dilated pupil, and hence incident on the retina at an angle with the normal, produce no abnormally high pupillomotor effect and $(b)$ that for practical purposes the part of the pupil through which light enters the eye is not a factor in determining pupil size.

Acknowledgment. The work described above was carried out in the Light Division of the National Physical Laboratory on behalf of the Chief of the Royal Naval Scientific Service, by whose permission this paper is published. 


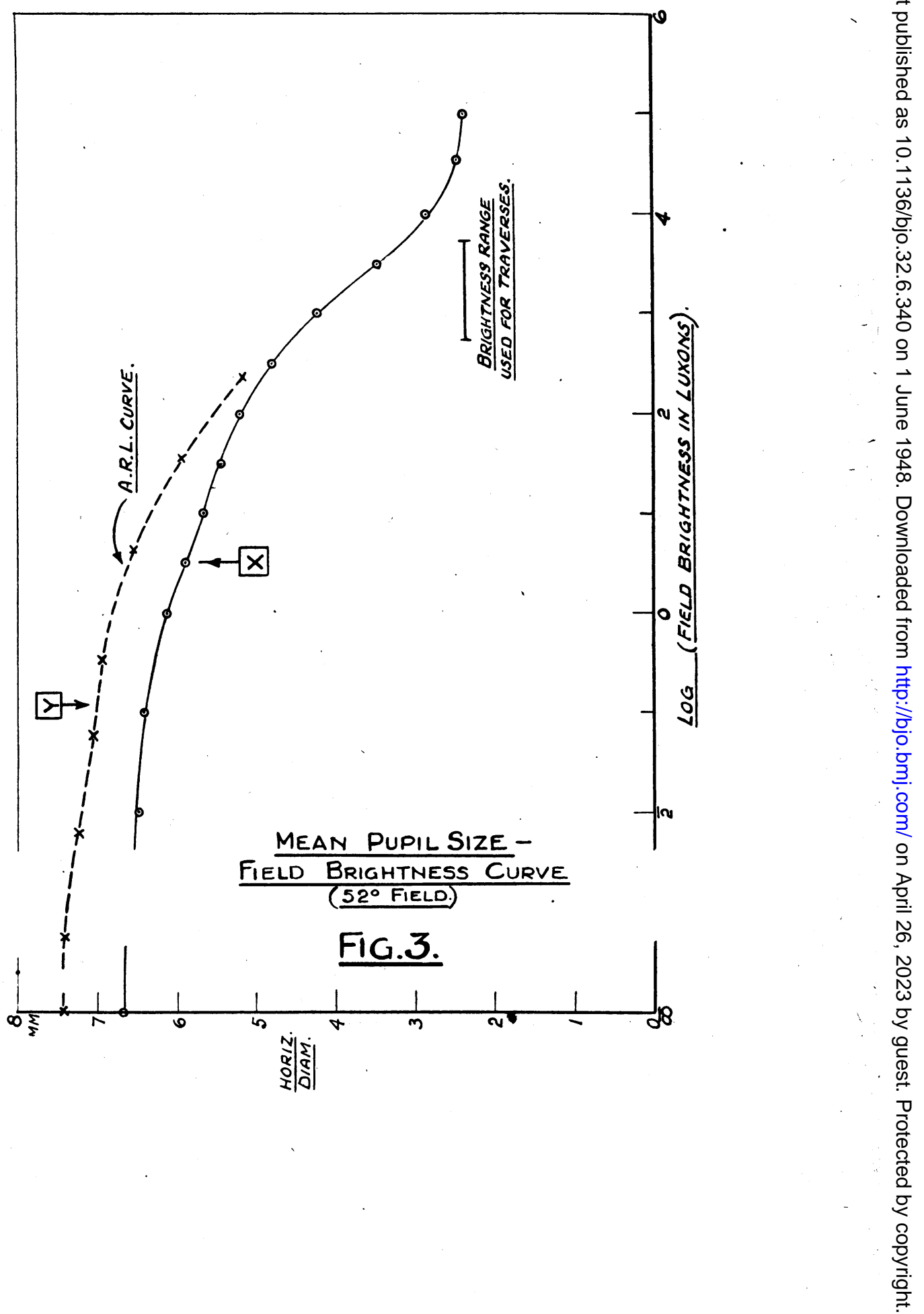



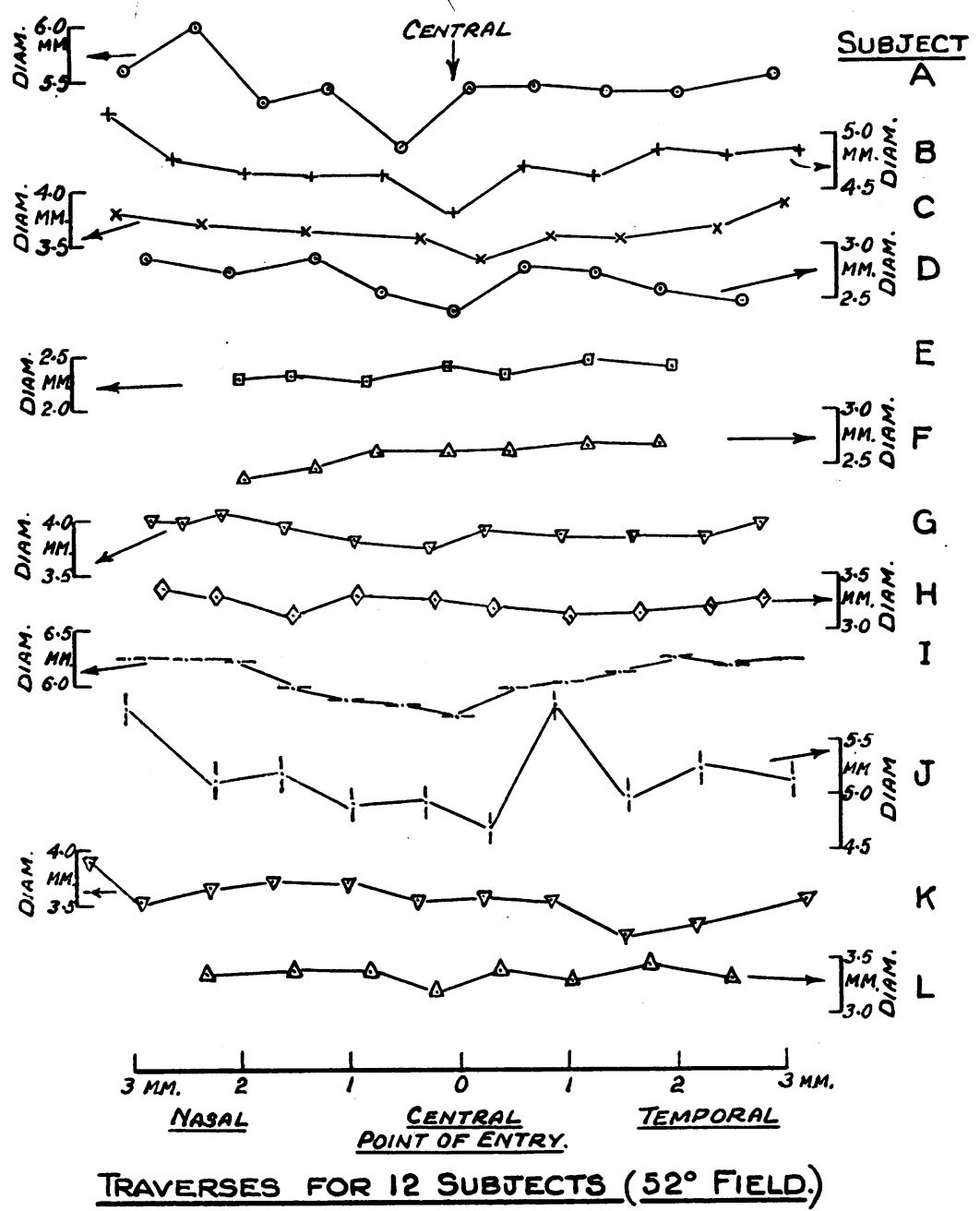

Fig. 4.

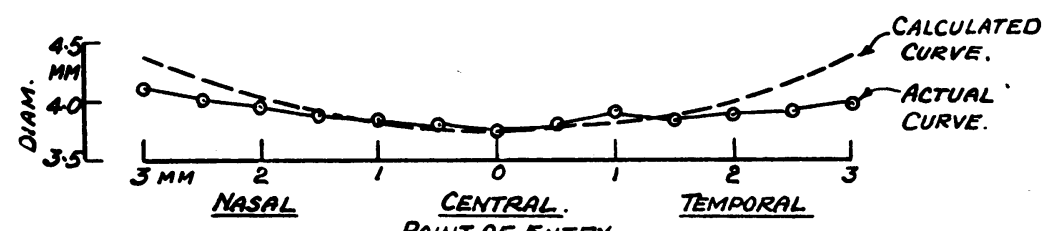

POINT OF ENTRY.

Calculated, and MEAN EXPERIMENTAL,CURVES.

FIG. 5. 